\title{
New solid state phosphate sensitive electrodes for routine environmental monitoring
}

\author{
Fikru Tafesse \\ Chemistry department, University of South Africa, \\ UNISA, Pretoria, South Africa \\ e-mail: Tafesf@unisa.ac.za
}

\begin{abstract}
Highly selective and sensitive phosphate sensors have been fabricated by constructing a solid membrane disk consisting of variable mixtures of aluminium powder $(\mathrm{Al})$, aluminium phosphate $\left(\mathrm{AlPO}_{4}\right)$ and powdered copper $(\mathrm{Cu})$. Both binary and ternary electrode systems are produced. The ternary membranes exhibit greater selectivity over a wide range of concentrations. The ternary electrode with the composition $25 \% \mathrm{AlPO}_{4}, 25 \% \mathrm{Cu}$ and $50 \% \mathrm{Al}$ was selected as our preferred electrode. The newly fabricated ternary membrane phosphate selective electrodes exhibited linear potential response in the concentration range of $1.0 \times 10^{-1}$ to $1.0 \times 10^{-6} \mathrm{~mol} \mathrm{~L}{ }^{-1}$. The electrodes also exhibit a fast response time of $<60 \mathrm{~s}$. Their detection limit is lower than $1.0 \times 10^{-6} \mathrm{~mol} \mathrm{~L}^{-1}$. The unique feature of the described electrodes is their ability to maintain a steady and reproducible response in the absence of an ionic strength control. The electrodes have a long lifetime and can be stored in air when not in use.
\end{abstract}

Keywords - Phosphate selective electrodes; selectivity coefficient; ionic strength; inorganic phosphate, environmental monitoring.

\section{Phosphates in the environment}

Phosphate rock is a non-renewable natural resource, mainly found in sedimentary and igneous deposits. Weathering and erosion of rocks gradually releases phosphorus as phosphate ions which are soluble in water. Most of the phosphate is washed into the natural waters from leaching processes. Plants and algae utilize phosphates as a nutrient for growth. Stunted and excess growth of plants and algae has been attributed to deficiency and surplus of phosphate ion respectively. When plant materials and waste products decay through bacterial action, the phosphate is released and returned to the environment for reuse. Humans have drastically altered the phosphorus cycle in many ways which includes the cutting of tropical rain forests, the radical use of phosphate containing food additives and the use of phosphate containing products like fertilizers and detergents. Rainforest ecosystems are supported primarily through the recycling of nutrients, with little or no nutrient reserves in their soils. As the forest is cut and/or burned, nutrients originally stored in plants and rocks are quickly washed away by heavy rains, causing the land to become unproductive. Agricultural runoff provides much of the phosphate found in waterways. Crops often cannot absorb all of the fertilizer in the soils, causing excess fertilizer runoff and increasing phosphate levels in rivers and other bodies of water. In aquatic systems, phosphates are found in dissolved, colloidal and particulate fractions as inorganic or organic compounds which may be biotic or abiotic particles [1]. Their concentrations in natural waters fluctuate with changes in physico-chemical conditions and biological activities. Seasonal changes in $\mathrm{pH}$, dissolved carbon dioxide and total dissolved calcium concentration do affect its availability [2]. It is very important to mention that phosphates and its derivatives also find wide applications in pharmaceutical industries, food industries, lasers and sensor technologies[3-6] to mention but a few. The need to regulate phosphate use and monitor its concentration in the environment cannot be overstated.

The need for a simple phosphate sensitive electrode that will be an alternative probe to the traditional colorimetric phosphate analysis is of a paramount importance. Ion selective electrodes are generally based on highly selective ionophores embedded in hydrophobic membranes. They are usually contacted with aqueous electrolytes or conductive wire and an internal or external reference. Analyte recognition process takes place followed by the conversion of chemical information into an electrical or optical signal. The ionophore is primarily responsible for the ion selectivity of the sensor by selectively and reversibly binding the analyte of interest. With optimized membrane formulations, the measured zero-current membrane potential can be directly related to the free ion activity of the analyte in the sample [7]. This research looks at the fabrication of a suitable ion selective electrode that will circumvent most of the difficulties encountered in the analysis of phosphates in environmental samples.

\section{Design and fabrication of the new solid state phosphate selective electrodes}

One approach towards a maintenance-free, robust and reliable sensor is to eliminate the inner filling solution and create an all-solid-state electrode [8]. The traditional barrel configuration of conventional electrodes (with internal filling solution) can prove cumbersome for some applications, so 
attempts at miniaturization brought about some new sensing systems, namely solid contact (SC) electrodes; such as solid crystal membranes, conducting filled polymer electrodes, and coated wire electrodes (CWE). This move to the total elimination of the internal filling solution of the conventional electrodes to the all-solid-state electrodes provides advantages such as; (a) simplicity of design, (b) mechanical flexibility, i.e. the electrode can be used horizontally, vertically, or inverted, and (c) the possibilities of miniaturization and micro fabrication. Also, the liquid (plasticizer) in solvent polymeric membranes may leach out and limit the robustness of such electrodes. The ionic conductivity is provided by a solid contact (SC) layer having mixed ionic and electronic conductivity between the inner reference element and the sensing membrane. A good example is the all-solid-state sodium-selective electrode based on a calixarene ionophore in a poly-vinyl chloride membrane with a polypyrrole solid contact [9]. This configuration is in contrast to typical membrane usage in which electrolyte solutions are in contact with opposite membrane sites.

\section{Experimental section}

All reagents used were either analytical reagent grade or the purest available commercially and were used without further purification. A digital screen Metrohm-781 pH/ion meter was used for potential measurements. The meter is capable of multi-point calibration with up to nine buffers (samples). Ion measurement can be by direct measurement or automatic standard addition or subtraction. Our fabricated phosphate selective electrodes were utilized as our indicator electrodes. In order to measure the change in potential difference across the ion-selective membrane as the ionic concentration changes, it is necessary to include in the circuit a stable reference voltage which acts as a half-cell from which the relative deviations is measured. The reference electrode utilized was a Metrohm silver/silver chloride $(\mathrm{Ag} / \mathrm{AgCl})$ double junction reference electrode. The reference electrode utilizes $3 \mathrm{M}$ potassium chloride $(\mathrm{KCl})$ as its internal solution. The $\mathrm{pH}$ values of the reacting solutions were monitored with a Metrohm Aquatrode plus pH electrode. All measurements were performed with the electrodes immersed in solutions and kept at ambient room temperature $\left(25 \pm 3^{\circ} \mathrm{C}\right)$. The solutions were slowly stirred with a magnetic stirrer.

\section{FABRICATION OF SOLID STATE MEMBRANE}

The novel feature of the present solid state phosphate ion selective electrode is the use of Aluminium phosphate $\left(\mathrm{AlPO}_{4}\right)$, Aluminium powder $(\mathrm{Al})$ and powdered copper $(\mathrm{Cu})$ mixed in various proportions as the membrane components. The various mixtures were grounded in mortar and pestle and transferred into a die. The die was placed in a Paul Weber 30 hydraulic press attached to Edward (EDM2) high vacuum suction pump. The time allowed for each pellet formation was 20 minutes and the pressure of the press was set at 7,000 atmospheres $\left(5.32 \times 10^{6} \mathrm{mmHg}, 709,275 \mathrm{kPa}\right)$. A DMM 15XP-A multimeter was used to measure electrical resistance across the thickness of each membrane. The pellets obtained were grouped into binary pellets (those that contain only two of the membrane components) and ternary pellets (those that contain the three membrane components). Fifteen (15) different membrane pellets were prepared in all, while each composition was made in triplicates. The mass of the membranes for all compositions is $0.5 \mathrm{~g}$. The binary membranes $\left(\mathrm{AlPO}_{4}+\mathrm{Al}\right.$ and $\left.\mathrm{AlPO}_{4}+\mathrm{Cu}\right)$ showed similar physical and electrical characteristics in terms of pellet thickness and electrical resistance. The membrane thickness and electrical resistance increased as the amount of aluminium phosphate was increased. It is observed that the binary membranes containing high amount of aluminium and copper are fairly strong with low electrical resistance. The ternary membranes $\left(\mathrm{AlPO}_{4}+\mathrm{Cu}+\mathrm{Al}\right)$ depicted comparable membrane thickness and electrical resistivity. The different membrane pellets were removed and stored in air and moisture controlled environment.

\section{ELECTRODE CONSTRUCTION}

Each electrode consists of a membrane pellet which is about $13.55 \mathrm{~mm}$ in diameter and varying thickness is fixed to a glass test tube of about $13 \mathrm{~mm}$ diameter and $100 \mathrm{~mm}$ length. A $99.9 \%$ pure copper wire of $1 \mathrm{~mm}$ diameter and $200 \mathrm{~mm}$ length was attached to the membrane with an epoxy resin. To obtain an air tight sealing, the epoxy resin was applied to the edges of the tube from the inside and the outside. The copper wire protruded at the other end of the test tube. The loose end of the copper wire was then connected to an electromotive force (EMF) meter.

\section{PROTOCOL FOR ELECTRODE USE}

$100 \mathrm{~mL}$ of the standard solutions $\left(1 \times 10^{-6} \mathrm{M}\right.$ to $\left.1 \times 10^{-1} \mathrm{M}\right)$ were put in a beaker and the electrode potential values measured. The test phosphate selective electrode and a reference electrode (both connected to an ion meter) were inserted in the slowly stirred phosphate solution of known concentration, $\mathrm{pH}$ and temperature. The observed potential value displayed on the ion meter digital screen was recorded. The response time of the electrode was also noted and the potential value was only taken when the reading was stable. The calibration curves were obtained by plotting the potential readings (in millivolt, $\mathrm{mV}$ ) against the $\log$ of phosphate activity $*[\mathrm{Pi}]$ (denoted in the graphs as $\log$ A). A calibration curve was obtained for each electrode composition. The [Pi] responses were obtained in triplicates and the average value was used for the calibration curve. The electrodes are immersed in $1 \times 10^{-3} \mathrm{~mol} \mathrm{~L}^{-1}$ solution of $\mathrm{Na}_{2} \mathrm{HPO}_{4} 7 \mathrm{H}_{2} \mathrm{O}$ for about 6 hours prior to use. The electrodes have been in use for about 3-4 times a week and 
have not deviated from their regression value in the past 12 months. The surface of the electrodes was polished with a soft paper before conditioning.

*[Pi] implies all phosphate species $\left(\mathrm{H}_{2} \mathrm{PO}_{4}{ }^{-1}\right.$ and $\left.\mathrm{HPO}_{4}^{-2}\right)$

\section{Results and discussion}

\section{CALIBRATION CURVE}

The results for the calibration curve studies for the phosphate sensitive electrode with a membrane formulation of $25 \% \mathrm{AlPO}_{4}, 25 \% \mathrm{Cu}$ and $50 \% \mathrm{Al}$ is given in figure 1.

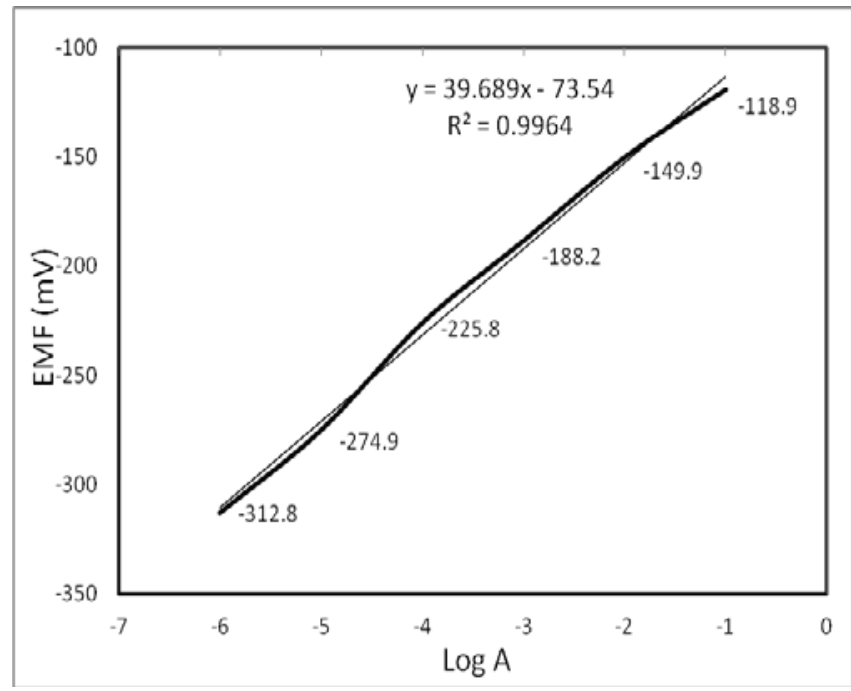

Figure 1. calibration curve for the ternary membrane electrode with a composition of $25 \% \mathrm{AlPO}_{4}, 25 \% \mathrm{Cu}$ and $50 \% \mathrm{Al}$.

The average Nernestian slope for this electrode was calculated as $39.689 \pm 1.399$. The complete Nernst equation is composed of Nernst factor, sensitivity factor and selectivity factor. If one does not consider the sensitivity and selectivity factors (i.e. assuming $100 \%$ sensitivity and selectivity), the Nernst equation can be written as: $\mathrm{E}=\mathrm{Eo}+\mathrm{RT} / \mathrm{nF}$. The equation $\mathrm{R} / \mathrm{F}$ can be replaced by 0.198 called the Nernst factor. The factor $0.198 \mathrm{~T} / \mathrm{n}$ will ideally give a value of about $59 \mathrm{mV}$ for a monovalent ion. The Nernst equation can be modified by the sensitivity of the electrode, $\mathrm{S} / 100 \%$. Similarly the selectivity of an electrode is never $100 \%$. Other ions may interfere. Hence selectivity factor has to be included to account for the interfering ions. Our ion selective electrodes are able to detect both the monohydrogen and dihydrogen phosphate ions which exist in the solution in accordance with the speciation diagram. Hence the value of $\boldsymbol{n}$ in the Nernst equation may be calculated by considering the concentration of the two major species that exist in the solution. For example, at a $\mathrm{pH}$ of $7.0,62 \%$ of the dihydrogen phosphate and $38 \%$ of the monohydrogen phosphate anions are presumed to exist in the solution giving rise to a calculated $\boldsymbol{n}$ value of about 1.4. Hence, the ideal Nernstian slope is expected to be $0.198 \mathrm{~T} / 1.4$ which produces a value of $42 \mathrm{mV}$. The Nernstian value obtained from the calibration curve reasonably agrees with the theoretical value.

\section{INTERFERENCE STUDIES}

All ion sensitive electrodes are sensitive to some other ions to some extent. For many applications these interferences are insignificant (unless there is a high ratio of interfering ion to primary ion) and can often be ignored. In some extreme cases, however, the electrode is far more sensitive to the interfering ion than to the primary ion and can only be used if the interfering ion is only present in trace quantities or even completely absent. Hence it is important to study the effects of all other ions in the medium. The response of an ion selective electrode is the potential developed as a function of the ionic activity of the species in solution. Hence when activity increases the electrode potential is expected to become more positive if the electrode is sensing a cation and more negative if it is sensing an anion. Figures 2 and 3 summarize the interference effects of some common cations and anions with varying concentration on a fixed $1 \times 10-2$ molar solution of phosphates at pH 7.0 and ambient temperature.

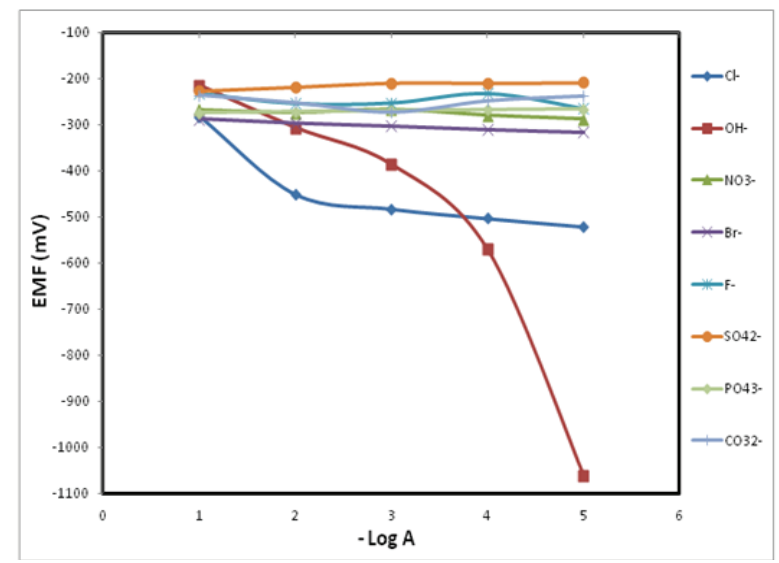

Figure 2. Interference effects of some common anions in the measurement of phosphates 


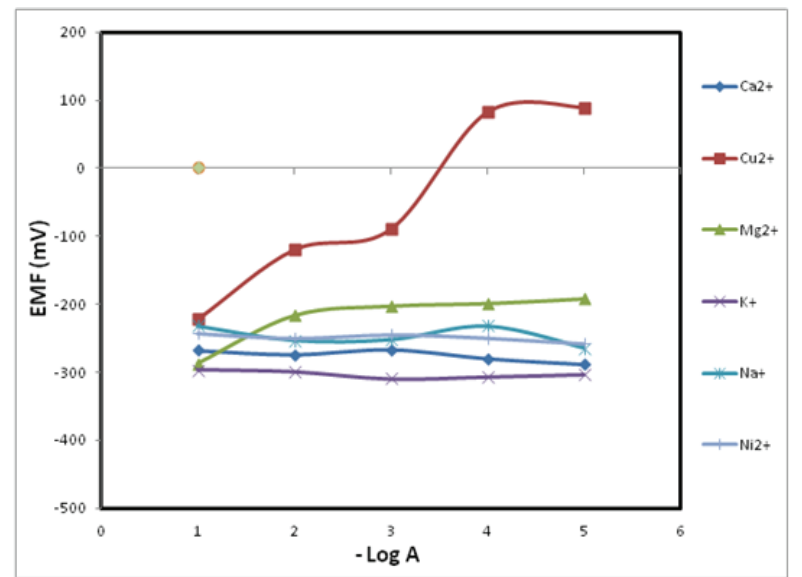

Figure 3. Interference effects of some common cations in the measurement of phosphates

The electrode is relatively insensitive to most anions and cations but responds well to anionic solutions containing chlorides and hydroxides. Cationic interferences were also investigated and it was found that this electrode responds well to $\mathrm{Cu}^{2+}$ ions as expected. Previous investigators [10,11] have employed the use of total ionic strength adjustment buffers (TISAB) to keep the ionic strength of the reacting solutions constant. In most cases they use $0.1 \mathrm{M} \mathrm{NaNO}_{3}$. The effect of a change in ionic strength brought about by the concentrations of phosphate species in our working solution is evident. However, the effect of this change on the total electrode potential was found to be less important at solutions concentration less than $1 \times 10^{-2} \mathrm{~mol} / \mathrm{L}$. Also, other TISAB formulations contain appreciable amounts of hydroxide which will interfere in phosphate ion determination with our electrode system. Hence in this study, no attempt was necessitated to keep the ionic strength constant as the main aim of our investigation is to produce robust phosphate selective electrodes that may be used in environmental samples without the need for ionic strength control. It should be worth mentioning that the determination of free phosphate in the presence of pyrophosphate is possible using our fabricated electrodes, as there was no observed interference.

\section{Acknowledgment}

This work was supported in part by grants from NRF-IRDP and graduate research and fellowship funds from the chemistry department, university of South Africa. I gratefully acknowledge Mr Martin Enemchukwu for the experimental work and assistance rendered. The Ecotoxicology flag ship project in the Department of Chemistry, UNISA, is also acknowledged

\section{REFERENCES}

[1] K. Robards, I. D. Mckelvie, R. L. Benson, P. J. Worsfold, N. J. Brundell, H. Casey; Determination of carbon, phosphorus, nitrogen and silicon species in water. Anal. Chim. Acta. 287 (1994) 147-190.

[2] G. Hanrahan, M. Gledhill, W. A. House, P. J. Worsfold; Evaluation of phosphorus concentrations in relation to annual and seasonal physico-chemical water quality parameters in a UK chalk stream. Water Res., 37(15) (2003) 3579-3589

[3] L. Cisse, T. Mrabet; World phosphate production: Overview and prospects. Phosphorus Research Bulletin, 15 (2004) 21-25.

[4] R. S. Malek, R. S. Kuntzman, D. M. Barret; Photosensitive potassium-titanyl phosphate laser vaporization of the benign obstructive prostate: observations on long term outcomes. J. Urol. 174, 4(1) (2005) 1344-1348.

[5] S. M. Yarema, D. Milam; Gain saturation in phosphate laser glasses. IEEE journal of Quantum Electronics. QE-18(11) (1982) 1941-1946.

[6] S. Jiang, J. D. Myers, D. L. Rhonehouse, M. Myers, R. E. Belford, S. J. Hamlin, R. E. Belford; Laser and thermal performance of a new Erbium doped phosphate laser glass. SPIE Vol. 2138, longer wavelengths lasers and applications (1994) 1-5.

[7] E. Bakker, E. Pretsch; Potentiometry at trace levels. Trends in analytical chemistry, 20(1), (2001) 11-19.

[8] D. P. Quan, L. T. Duan, C. X. Quang, P. H. Viet; A conducting polymer based solid-contact potassium selective electrode. Analytical Sciences. Vol. 17 Supplement (2001) i749-i752.

[9] A. Cadogan, Z. Gao, A. Lewenstam, A. Ivaska; All-SolidState-Sodium-Selective electrode based on a calixarene ionophore in a poly(vinyl chloride) membrane with a polypyrrole solid contact. Anal. Chem. 64 (1992) 2496-2501.

[10] G. Somer, S. Kalayci, I. Basak; Preparation of a new solid state fluoride ion selective electrodes and application. Talanta, 80 (2010) 1129-1132.

[11] G. Ekemekci, S. Kalayci, G Somer; A solid state hydroxide ion selective electrode for the measurement of high pH values. Sensors and Actuators, B 101, (2004) 260-264. 